\title{
Analytical Assessment of Stochastic Spread of Demand for the Port Storage Capacity
}

\author{
A.L. Kuznetsov \\ Admiral Makarov State University of Maritime and Inland Shipping, Saint-Petersburg, Russia
}

\author{
H. Oja \& A.D. Semenov \\ Konecranes Finland Corp., Hyvinkää, Finland
}

\begin{abstract}
At design stages of any sea port development projects one of the key tasks is to estimate the amount of cargo volume to be stored on the port warehouse. The shortage of the warehouse facilities would disrupt port operations and affect the port marketing position, while the surplus capacity would raise the selfcost of the services rendered by the port. Many port developing projects and long years of operational practice have resulted into certain commonly accepted mathematical techniques that enable to assess all main parameters sufficiently accurately. With ever-growing completion between the ports worldwide, to find a delicate balance between the cost and quality becomes a core task behind nearly every aspect of port design activity. The tools that have been used for centuries in port design and development started to lose their adequacy in modern economic and logistic environment. As the response for this challenge the port designers more and more move to simulation models. In the same time, an adequate simulation models need not only accurate and reliable data, but also requests quite long time. Moreover, the models of the kind usually are created ad hoc, reflecting particular features of the primal object under development and forfeiting the generality and universality of analytical models. At beginning stages of port developing one need to have simple and easy tools for the preliminary accession of project parameters, since usually there are several variants and the full-scaled simulation of them is excluded. Still, these tools should be more enhanced sophisticated than common analytical formulae. The main drawback of the formula calculation (streaming computing by the current IT terminology) is they principally deal with deterministic values, while the real worlds is inhibited with the stochastic ones. The study represented here is an attempt to narrow this gap. The area selected to demonstrate the approach is the port warehouse size, regardless of the cargo type handled. In the same time, this technique can be spread on many other port project parameters needed to be assessed.
\end{abstract}

\section{INTRODUCTION}

Every port consulting and design organization has its own carefully selected toolkit of port project and design techniques and know-how to use them efficiently. In the same time, all the designers face the very same problem: at early stages of the project it is not wise to use sophisticated and advanced tools that give accurate results, but simple tools permit to gain only rough estimations [1-3]. The methodological problem behind this contradiction is in the nature of the data used by these approaches. Simple and easy formula computations deal with the deterministic data, while the reality demands to take into account the fluctuations caused by the stochastic character of all main variables [4-6]. This paper offers an extension of the classical deterministic approach that will help to cover the gap between analytical and simulation approaches. Moreover, the results could turn to be 
useful in proving the adequacy of any simulation models, which itself is a very big problem.

\section{METHODS AND MATERIALS}

Let's consider the calculation of the average amount of cargo that dwells in the port $\mathrm{E}$, when we know the average duration of the cargo party formation (accumulat ion on the warehouse) $T_{f}$, mean interval of party arrivals (ship calls interval) $T_{i n t}$ and mean ship party's volume (call size) V . Under the assumption of triangular form of the party accumulation (i.e. with the constant rate of cargo arrival/departure on the terminal), within the one party's dwell time on the terminal $T_{d w}=\frac{T_{f}}{2} \quad$ there will be $\quad \frac{T_{d w}}{T_{i n t}}=\frac{1}{2} \frac{T_{f}}{T_{i n t}} \quad$ cargo parties of the volume $\mathrm{V}$ entering the warehouse, so the average total amount is $\quad E=\frac{1}{2} V \frac{T_{f}}{T_{i n t}}$.

All of variables in this formula are stochastic values, thus the resulting volume of the cargo storage is the stochastic one, too. What judgments on the character of the end value can we made?

For the sake of convenience let us make the substitution of the working variables, particularly $V=X, T_{f}=Y, \mathrm{Z}=\frac{1}{T_{\text {int }}}$. In this notation the formula will take the form of $E=\frac{1}{2} X Y Z$. Let us assume that we know the values of two main numeric characteristics of the values $\mathrm{X}, \mathrm{Y}, \mathrm{Z}$, specifically the mathematical expectations $M[X]=m_{x}$, $[Y]=m_{y} \quad, \quad M[Z]=m_{z} \quad$ and dispersions $D[X]=D_{x}, D[Y]=D_{y}, D[Z]=D_{z}$.

Let us compute the characteristics of the target computation value of the amount of storage, or $M[E]=m_{E}$ and $D[E]=d_{E}$.

The evaluation of the mean arithmetic values causes no difficulties since by the theorem on the computation of independent stochastic values we directly have

$M[E]=m_{E}=M\left[\frac{1}{2} \mathrm{XYZ}\right]=\frac{1}{2} M[\mathrm{XYZ}]$.
In other words, the mathematical expectations of cargo store amount is

$$
M[E]=\frac{1}{2} m_{x} m_{y} m_{z}
$$

The computation of the dispersion is a bit more laborious. Actually, let us denote $\mathrm{XYZ}=A$. Since for any non-stochastic value $c$ we have

$$
\mathrm{D}[\mathrm{cA}]=c^{2} \mathrm{D}[\mathrm{A}] \text {, then } \mathrm{D}\left[\frac{1}{2} \mathrm{~A}\right]=\frac{1}{4} \mathrm{D}[\mathrm{A}] \text {. }
$$

Further on, by the definition of dispersion we have $\mathrm{D}[\mathrm{XYZ}]=\mathrm{D}[\mathrm{A}]=M\left[\left(A-m_{A}\right)^{2}\right]$. Since the values $\mathrm{X}, \mathrm{Y}, \mathrm{Z}$ are independent, $m_{A}=m_{x} m_{y} m_{z} \cdot$ Respectfully

$$
\begin{aligned}
& \mathrm{D}[\mathrm{XYZ}]=M\left[\left(X Y Z-m_{x} m_{y} m_{z}\right)^{2}\right]= \\
& =M\left[X^{2} Y^{2} Z^{2}\right]-2 m_{x} m_{y} m_{z} M[\mathrm{XYZ}]+m_{x}^{2} m_{y}^{2} m_{z}^{2}
\end{aligned}
$$

With independent $X, Y, Z$ the values $X^{2}, Y^{2}, Z^{2} \quad$ also are independent, so $M\left[X^{2} Y^{2} Z^{2}\right]=M\left[X^{2}\right] M\left[Y^{2}\right] M\left[Z^{2}\right] \quad$ and $M[\mathrm{XYZ}]=m_{x} m_{y} m_{z}$ and further

$$
\mathrm{D}[\mathrm{XYZ}]=M\left[X^{2}\right] M\left[Y^{2}\right] M\left[Z^{2}\right]-m_{x}^{2} m_{y}^{2} m_{z}^{2}
$$

In the same time, $M\left[X^{2}\right]$ is the second initial moment of the stochastic value $X$, so it could be expressed through the dispersion as $M\left[X^{2}\right]=D[X]+m_{x}^{2} \quad$. Similarly, $M\left[Y^{2}\right]=D[Y]+m_{y}^{2}$ and $\left[Z^{2}\right]=D[Z]+m_{z}^{2}$.

If inserted in the received formulae, these expressions will give

$$
\begin{aligned}
& \mathrm{D}[\mathrm{XYZ}]=M\left[X^{2}\right] M\left[Y^{2}\right] M\left[Z^{2}\right]-m_{x}^{2} m_{y}^{2} m_{z}^{2}= \\
& \left(D[X]+m_{x}^{2}\right)\left(D[Y]+m_{y}^{2}\right)\left(D[Z]+m_{z}^{2}\right)-m_{x}^{2} m_{y}^{2} m_{z}^{2}= \\
& D_{x} D_{y} D_{z}+m_{x}^{2} D_{y} D_{z}+D_{x} m_{y}^{2} D_{z}+D_{x} D_{y} m_{z}^{2}+m_{x}^{2} m_{y}^{2} D_{z}+ \\
& +m_{x}^{2} D_{y} m_{z}^{2}+D_{x} m_{y}^{2} m_{z}^{2}
\end{aligned}
$$

Eventually, the seeking dispersion of the total amount of cargo stored at a terminal could be expressed as 


$$
\begin{aligned}
& \boldsymbol{D}_{\boldsymbol{E}}=\frac{1}{4}\left(\boldsymbol{D}_{x} \boldsymbol{D}_{\boldsymbol{y}} \boldsymbol{D}_{z}+\boldsymbol{m}_{x}^{2} \boldsymbol{D}_{\boldsymbol{y}} \boldsymbol{D}_{z}+\boldsymbol{D}_{\boldsymbol{x}} \boldsymbol{m}_{y}^{2} \boldsymbol{D}_{z}+\boldsymbol{D}_{\boldsymbol{x}} \boldsymbol{D}_{y} \boldsymbol{m}_{z}^{2}\right. \\
& \left.+\boldsymbol{m}_{x}^{2} \boldsymbol{m}_{y}^{2} \boldsymbol{D}_{z}+\boldsymbol{m}_{x}^{2} \boldsymbol{D}_{\boldsymbol{y}} \boldsymbol{m}_{z}^{2}+\boldsymbol{D}_{\boldsymbol{x}} \boldsymbol{m}_{y}^{2} \boldsymbol{m}_{z}^{2}\right)
\end{aligned}
$$

The standard deviation of this value is $\delta_{E}=\sqrt{\boldsymbol{D}_{\boldsymbol{E}}}$.

\section{RESULTS AND DISCUSSION}

We could expect by the central limit theorem (stating that the sum of many weakly interdependent values has a distribution close to the normal one) that the stochastic value of total amount of cargo stored at a terminal has the Gaussian distribution [7]. Since we managed to assess the values of its mathematical expectation and dispersion (standard deviation), we could construct the correspondent cumulative distribution function, as Fig. 1 shows.

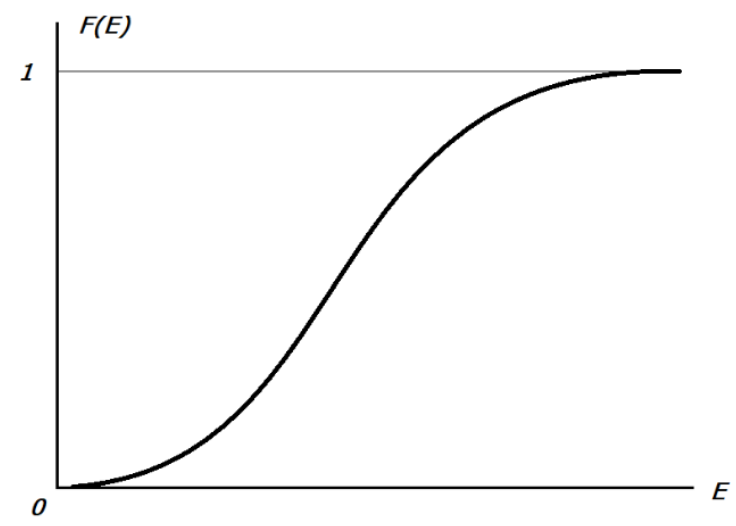

Figure 1. Gaussian cumulative distribution function

By the definition, the cumulative distribution function is the probability of the event $e<E$, where $e$ is a current variable. In the context of our study, we could interpret this function as the probability that the warehouse of the size $e$ would be sufficient to contain the required amount of cargo [8-9]. If the size is equal to the mathematical expectations, in $50 \%$ it will be enough and in $50 \%$ there will be the shortage of store facilities.

Knowing the properties of Gaussian distribution, we could expect also, that the interval $\left[m_{E}-2 \cdot \delta_{E}, m_{E}+2 \cdot \delta_{E}\right]$ holds around $95 \%$ of all values, thus the warehouse with the size $m_{E}+2 \cdot \delta_{E}$ will be insufficient only in 2,5\%, as Fig. 2 shows.

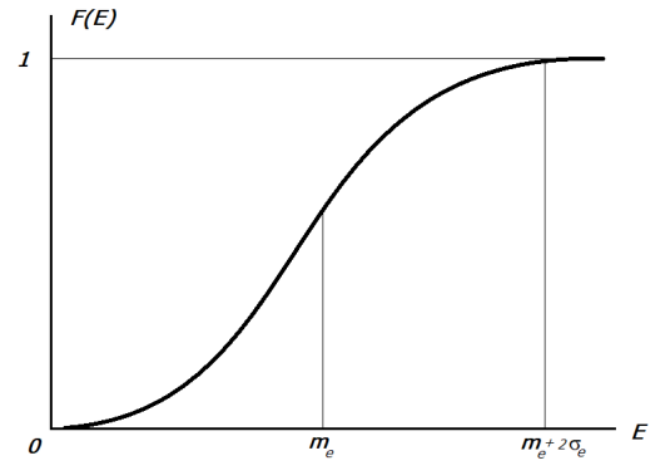

Figure 2. Assessment of the required warehouse size

In order to prove the correctness of the results, the simulation experiments were conducted. Fig. 3 shows the results of an experiment of this simulation, with vessels arrival intervals, party sizes and dwell times distributed by Gaussian distribution.

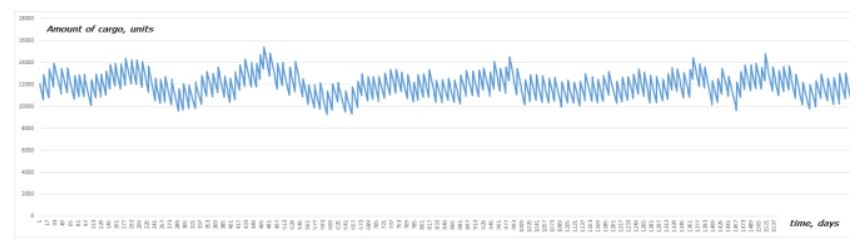

Figure 3. Simulation of the warehouse dynamics

The values of the referenced parameters are represented by the tab. 1 .

Table 1. The referenced parameters of simulation

\begin{tabular}{|c|c|c|c|c|}
\hline Description & Notation & Units & $\boldsymbol{M}_{\boldsymbol{x}}$ & $\boldsymbol{\sigma}_{\boldsymbol{x}}$ \\
\hline Cargo party & $\mathrm{V}$ & {$[$ units] } & 2400 & 240 \\
\hline Formation time & $\mathrm{T}$ & [hours] & 120 & 12 \\
\hline Arrival interva & $\mathrm{t}$ & {$[$ hours] } & 12 & 2 \\
\hline
\end{tabular}

The results of the statistical processing (i.e. the experimental distribution of values shown by Fig. 3, are displayed on Fig. 4 a) and b).
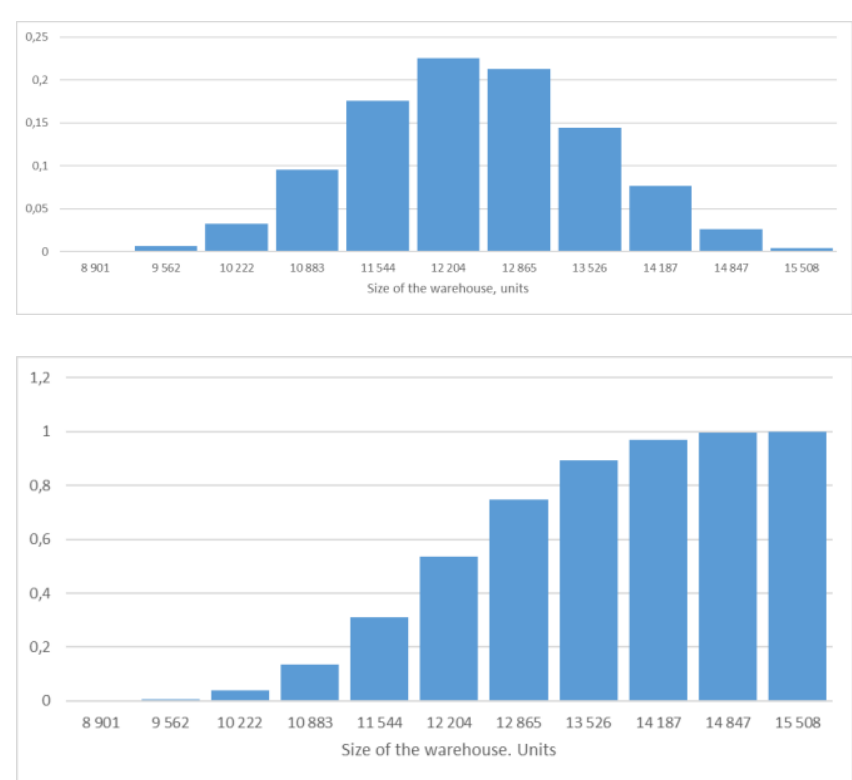

Figure 4. Distribution of the simulated values

Density of distribution b) Cumulative distribution function 
The calculation by the technique described in this study gives the characteristics of the final distribution shown by the Tab. 2 .

Table 2. Calculated parameters of simulation

\begin{tabular}{|c|c|c|c|}
\hline Description & Notation & Units & Value \\
\hline Math.expectation of $\mathrm{E}$ & $\mathrm{M}_{\mathrm{E}}$ & [units] & 12014 \\
\hline Dispersion of $\mathrm{E}$ & $\mathrm{D}[\mathrm{XYZ}]$ & [units $^{2}$ ] & 9012302 \\
\hline Standard deviation & $\sigma$ & [units] & 3002 \\
\hline Spread & $2^{*} \sigma$ & [units] & 6004 \\
\hline Upper limit & $\mathrm{M}_{\mathrm{E}}+2^{*} \sigma$ & [units] & 18018 \\
\hline
\end{tabular}

The calculated mathematical expectation and dispersion enables to build the model cumulative distribution function (Fig. 5) which practically coincides with the one produced by the processing of the simulation data shown by Fig. 4 .

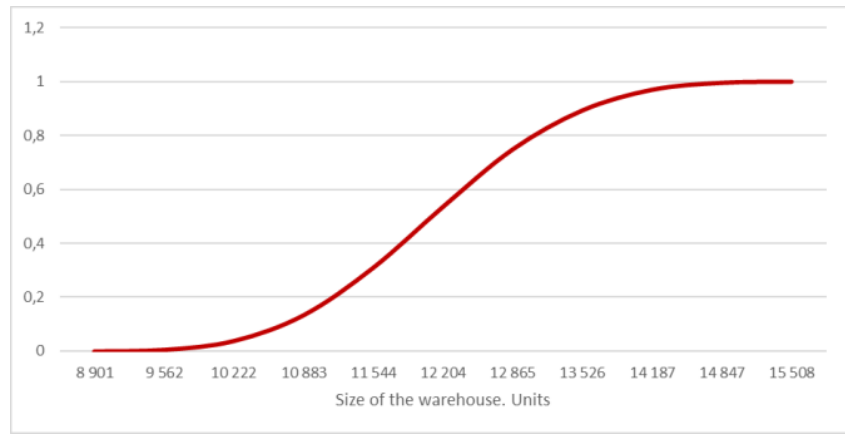

Figure 5. Reconstructed cumulative distribution function of the warehouse size

The proximity of these functions was confirmed by statistically valid amount of experiments with different distributions (some of them even nonGaussian), thus enabling to state that this simple technique is adequate. The designer would only make several reasonable assumptions over the distribution of the input values and immediately could see the spread of the output values. Maybe not for $100 \%$ reliable, this method could find a proper slot in the port designer's toolkit.

\section{CONCLUSIONS}

1 The analytical (formula) calculations cannot give the perception of the values' spread over mean values.

2 The full-scaled simulation could bring the desired results but at the cost of developing laborious procedures not justified on the beginning stages of the port projects.

3 The method proposed in this study enables to receive a reasonable estimation of the stochastic values by rather small extension of common formula deterministic technique.

4 The method does not take into account any specific properties of the cargo and thus could be used for all types of port warehouses.

5 The approach described in the paper using the example of the warehouse could be extended to cover the assessments of other technological parameters treated as stochastic values.

\section{REFERENCES}

[1] UNCTAD. Port development: A handbook for planners in developing countries. - UN, 1978.

[2] Thoresen C. A. Port designer's handbook. - Thomas Telford Ltd, 2010.

[3] Böse J. W. et al. Handbook of terminal planning. - New York: Springer, 2011. - V. 49. - P. 433.

[4] Kuznetsov A. L., Kirichenko A. V., Eglit J. J. Simulation Model of Container Land Terminals //TransNav: International Journal on Marine Navigation and Safety of Sea Transportation. - 2018. - V. 12. - №. 2.

[5] Kuznetsov A. L., Kirichenko A. V., Slitsan A. E. Simulation for assessment of bulk cargo berths number //IOP Conference Series: Earth and Environmental Science. - IOP Publishing, 2017. - V. 87. - №. 6. - P. 062010

[6] Kuznetsov A. L., Galin A. V. Port development simulation I/MARINE INTELLECTUAL TECHNOLOGIES. - 2018. - V. 1. - №. 3. - P. 176-182.

[7] Chung K. L., Zhong K. A course in probability theory. Academic press, 2001

[8] Hammersley J. Monte Carlo methods. - Springer Science \& Business Media, 2013.

[9] Klenke A. Probability theory: a comprehensive course. Springer Science \& Business Media, 2013. 